\title{
Dynamic Complexity of a Switched Host-Parasitoid Model with Beverton-Holt Growth Concerning Integrated Pest Management
}

\author{
Changcheng Xiang, ${ }^{1,2,3}$ Zhongyi Xiang, $^{1}$ and Yi Yang ${ }^{2}$ \\ ${ }^{1}$ College of Forestry and Horticulture, Hubei Minzu University, Enshi 445000, China \\ ${ }^{2}$ College of Science, Hubei Minzu University, Enshi 445000, China \\ ${ }^{3}$ Key Laboratory of Biologic Resources Protection and Utilization, Hubei Minzu University, Enshi, Hubei 445000, China
}

Correspondence should be addressed to Changcheng Xiang; xcc7426681@126.com

Received 8 February 2014; Accepted 27 April 2014; Published 28 May 2014

Academic Editor: Bernard J. Geurts

Copyright (C) 2014 Changcheng Xiang et al. This is an open access article distributed under the Creative Commons Attribution License, which permits unrestricted use, distribution, and reproduction in any medium, provided the original work is properly cited.

\begin{abstract}
The switched discrete host-parasitoid model with Beverton-Holt growth concerning integrated pest management has been proposed, and the switches are guided by the economic threshold (ET). The integrated pest management (IPM) tactics are applied to prevent the economic injury if the density of host population exceeds the ET, and the IPM tactics are called off once the density of host population descends below ET. To begin with, the regular and virtual equilibria of switched system has been discussed by two or three parameter-bifurcation diagrams, which reveal the regions of different types of equilibria. Besides, numerical bifurcation analyses about inherent growth rates show that the switched discrete system may have complicated dynamics behavior including chaos and the coexistence of multiple attractors. Finally, numerical bifurcation analyses about killing rates indicate that the system comply with the Volterra principle, and initial values of both host and parasitoid populations affect the host outbreaks times.
\end{abstract}

\section{Introduction}

In recent years, integrated pest management (IPM) has been introduced as a long-term, more effective, and low-cost control strategy to reduce the pest damage which integrated with biological, cultural, and chemical tactics to control pest populations at the tolerable level $[1,2]$. Moreover, IPM has been proved to be effective pest control strategies through theory analysis and experiments [3]. It aims at alleviating the pollution of plants and animals due to the excessive use of pesticides. IPM mainly involves two important and essential components (chemical control and biology control). Chemical control (e.g., spaying pesticides) is an effective strategy to reduce the pest population [4]. However, longterm chemical tactics will lead to pest drug-resistance and environmental pollution, while biological control tactics are another important control strategy to overcome the deficiencies of chemical control. Biological control is mainly adopted by periodically releasing its natural enemies, such as predators, parasitoids, or pathogen to keep pest population below the given economic threshold (ET). The ET refers to the population density at which control action should be taken to prevent an increasing pest population from reaching the economic injury level (EIL), where EIL is defined as the lowest population density of a pest that will cause economic damage $[1,5,6]$.

Recently, many impulsive differential equation models with fixed pulse-type actions have been proposed to model the periodic IPM strategy by Liang and Tang $[7,8]$. Nevertheless, these impulsive differential equation models, however, have applied the periodic IPM strategy, but few considered the ET. Thus, the switching systems (or Filippov systems) with ET described by ODE model have been brought into ecology systems with IPM [9-12]. We should spray pesticides and release natural enemies if ET is approached but not reached the EIL. So far, few studies are proposed on the discrete switching model in ecology with IPM. For the reason, in this work, we will develop switched discrete model with IPM based on host-parasitoid model with Beverton-Holt growth. 
The main purpose of this paper is to construct the switched discrete host-parasitoid model with Beverton-Holt growth concerning integrated pest management (IPM), and the economic threshold (ET) is chosen to guide the switches. First of all, we establish a switching system to model the process of releasing natural enemies and spraying pesticides (or harvesting pest) guided by ET. The existence of several types of equilibria of switched system has been discussed briefly, and two or three parameter-bifurcation diagrams reveal the regions of different types of equilibria including regular and virtual equilibria. What is more, numerical bifurcation analyses show that the switched discrete system may have complicated dynamics behaviors including chaos and the coexistence of multiple attractors. We further address how the key parameters and initial values of both host and parasitoid populations affect the host outbreaks.

\section{Host-Parasitoid Model}

Now we consider the individuals of host (pest) population which undergo intraspecific competition; the well-known Beverton-Holt model takes into account intraspecific competition [13]

$$
H_{t+1}=\frac{\alpha H(t)}{b+H_{t}}, \quad t=1,2, \ldots,
$$

where $H_{t}$ is the population densities in generation $t(t=$ $0,1,2,3, \ldots), \alpha$ is an inherent growth rate, and $b$ is a constant.

To take into account the effect of parasitoid interference on the host population, the interaction between the host and the parasitoid is governed by (2) of difference equations [14]. From the perspective of pest control, the host population is regarded as a pest and the parasitoid population is treated as a natural enemy of the host population. The host-parasitoid (pest-enemy) interaction in this system does not consider the external enemy releasing and insecticide spraying:

$$
\begin{gathered}
H_{t+1}=\frac{\alpha H(t)}{b+H_{t}} e^{-\beta P_{t}}, \\
P_{t+1}=H_{t}\left(1-e^{-\beta P_{t}}\right),
\end{gathered}
$$

where $H_{t}$ is the host population densities and $P_{t}$ is the parasitoid population densities in generation $t(t=0,1,2,3, \ldots)$. If the host population represents the pests and parasitoid population denotes the natural enemies, then the model describes the pest-natural enemy interaction. Furthermore, the IPM strategies can be taken into account when we aim to control the pest population. Actually, the IPM tactics could only be implemented when the density of pest population exceeds the ET, and the control tactics must be suspended once its density falls below the ET. As a consequence, the switching host-parasitoid model concerning IPM is established based on system (2) as follows:

$$
\begin{gathered}
H_{t+1}=\frac{\alpha H(t)}{b+H_{t}} e^{-\beta P_{t}}, \quad H_{t}<\mathrm{ET}, \\
P_{t+1}=H_{t}\left(1-e^{-\beta P_{t}}\right), \\
H_{t+1}=(1-p) \frac{\alpha H(t)}{b+H_{t}} e^{-\beta P_{t}}, \quad H_{t} \geq \mathrm{ET}, \\
P_{t+1}=H_{t}\left(1-e^{-\beta P_{t}}\right)+\tau,
\end{gathered}
$$

where $p$ represents the proportion of pest population (host) which is killed or caught $(0<p<1)$, and $\tau$ represents the releasing numbers of the natural enemies (parasitoid). Particularly, if $p=0$ and $\tau>0$, then only the biological control measures are applied, and if $p>0$ and $\tau=0$, then only the chemical control tactics are applied. Moreover, we assume $p \tau \neq 0$, which means that at least one control measure is applied once density of pest population exceeds the ET. From the switching system (3), it is divided into two subsystems. One is free subsystem when $H_{t}<\mathrm{ET}$, and we denote it by $S_{G_{1}}$; the other is controllable subsystem when $H_{t} \geq \mathrm{ET}$, and we denote it by $S_{G_{2}}$, where $G_{1}=\left\{(H, P) \mid H_{t}<\right.$ ET, $H>0, P>0\}, G_{2}=\left\{(H, P) \mid H_{t} \geq\right.$ ET, $\left.H>0, P>0\right\}$.

\section{Equilibria of Two Subsystems}

Although the existence and stability of equilibria of subsystem $S_{G_{1}}$ have been investigated [15], we also prove the existence of the equilibria of two subsystems briefly, which are useful for discussing the types of the equilibria of the whole switching system (3).

3.1. Equilibria of the Subsystems $S_{G_{1}}$ and $S_{G_{2}}$. For subsystem $S_{G_{1}}$, let $H_{t}=H_{t+1}=H_{1 *}$ and $P_{t}=P_{t+1}=P_{1 *}$; then the equilibrium $\left(H_{1 *}, P_{1 *}\right)$ satisfies the following equations:

$$
H_{1 *}=\frac{\alpha H_{1 *}}{b+H_{1 *}} e^{-\beta P_{1 *}}, \quad P_{1 *}=H_{1 *}\left(1-e^{-\beta P_{1 *}}\right) .
$$

Notice that the $P$-component of an interior steady state $\left(H_{1 *}, P_{1 *}\right)$ must satisfy

$$
\alpha=(b+h(P)) e^{\beta P}>b+h(P),
$$

where $N=h(P)=P /\left(1-e^{-\beta P}\right)$ for $P>0$. Since $\lim _{P \rightarrow 0^{+}} h(P)=1 / \beta, h^{\prime}(P)>0$ for $P>0$ and $\lim _{P \rightarrow \infty} h(P)=$ $\infty$, we see that (5) has a positive solution $P_{1 *}$ if and only if

$$
\alpha>\frac{b+\beta}{\beta}, \quad \beta N_{*}>1 \text {. }
$$

Similarly, we can discuss the existence of equilibria of subsystem $S_{G_{2}}$. Note that $S_{G_{2}}$ has a boundary steady state $E_{+0}^{\prime}=(\tau, 0)$ if $\tau>0$, and the interior steady state $E_{2 *}=$ $\left(H_{2 *}, P_{2 *}\right)$ satisfies the following equations. For subsystem $S_{G_{2}}$

$$
\begin{aligned}
H_{2 *} & =(1-p) \frac{\alpha H_{2 *}}{b+H_{2 *}} e^{-\beta P_{2 *}}, \\
P_{2 *} & =H_{2 *}\left(1-e^{-\beta P_{2 *}}\right)+\tau .
\end{aligned}
$$


The interior steady state $\left(H_{2 *}, P_{2 *}\right)$ must satisfy

$$
(1-p) \alpha=(b+h(P)) e^{\beta P}>b+h(P),
$$

where $H=h(P)=(P-\tau) /\left(1-e^{-\beta P}\right)$ for $P>\tau$. Since $1-e^{-\beta P}<$ $\beta P$, let $h_{1}(P)=(P-\tau) / \beta P$, so

$$
(1-p) \alpha=(b+h(P)) e^{\beta P}>b+h_{1}(P) .
$$

Since $\lim _{P \rightarrow \tau^{+}} h_{1}(P)=0, h_{1}^{\prime}(P)>0$ for $P>\tau$ and $\lim _{P \rightarrow \infty} h_{1}(P)=1 / \beta$, we see that (8) has a positive solution $H_{2 *}$ if and only if $P_{2 *}>\tau$ and $\alpha>b /(1-p)$.

3.2. Equilibria for the Switching System (3). The different types of equilibria of Filippov systems or switching systems play a key role in analyzing the dynamical behavior and discussing the biological implications, which have been widely used in the evolution of biodiversity in multispecies systems [16], piecewise HIV virus dynamic model with $C D 4^{+} T$ cell count-guided therapy [17], and nonsmooth plant disease models with economic thresholds [18]. To show the equilibria of switching system (3), we also need the definition of regular equilibrium and virtual equilibrium about discrete switching system.

Definition 1. A point $Z_{*}=\left(H_{*}, P_{*}\right)$ is called a regular equilibrium of system (3) if $Z_{*}$ is an equilibrium of subsystem $S_{G_{1}}$, and $F\left(H_{*}\right)<$ ET or if $Z_{*}$ is an equilibrium of subsystem $S_{G_{2}}$, and $F\left(H_{*}\right) \geq$ ET, denoted by $E_{S_{G_{1}}}^{r}$ and $E_{S_{G_{2}}}^{r}$, respectively. A point $Z_{*}$ is called a virtual equilibrium of system (3) if $Z_{*}$ is an equilibrium of subsystem $S_{G_{1}}$, and $F\left(H_{*}\right) \geq$ ET or if $Z_{*}$ is an equilibrium of subsystem $S_{G_{2}}$, and $F\left(H_{*}\right)<$ ET, denoted by $E_{S_{G_{1}}}^{v}$ and $E_{S_{G_{2}}}^{v}$, respectively.

Since the system (3) is a transcendental equation and the interior equilibria of two subsystems cannot be solved analytically, we turn to employ the numerical methods to simulate the existence of different types of equilibria and coexistence. In what follows, we first choose $\alpha$ and ET as bifurcation parameters and fix all others as those in Figure 1(a). The results show that the parameter space has been divided into four regions. For example, when $\alpha \epsilon$ $[1.61 .85]$ and ET $\in[1.3,3]$, there does not exist any interior equilibria, as indicated in region I. Region II is marked in red, and there only exists $S_{G_{1}}^{v}$; if $\alpha \in$ [1.852.9], there exist two regions divided into region III (purple) and region IV (yellow). Moreover, in the purple regions $E_{S_{G_{1}}}^{r}$ and $E_{S_{G_{2}}}^{v}$ coexist. In the yellow region, $E_{S_{G_{1}}}^{v}$ and $E_{S_{G_{2}}}^{v}$ coexist. Note that the parameter regions (existences of equilibria) depend on the different values of $\alpha$. In order to design the optimal control strategy and to keep the density of pest population below ET, we should choose the appropriate parameters $\alpha$ and ET such that the interior equilibrium of $S_{G_{1}}$ subsystem and $S_{G_{2}}$ subsystem become virtual. Therefore, from the point of the IPM, region IV is the ideal region to design the optimal control strategy.

From the above discussion, we know that the equilibria are related to parameters $\alpha$ and ET. Moreover, the parameter $\beta$ has an effect on the equilibria. To show this, we let the parameter $\beta$ vary with the parameter $\alpha$ at the same time and the other parameters are shown in Figure 1(b). The results show that the area of four regions has great changes. For example, the area of region I (green) is enlarged several times and region III shrinks to a triangular area compared with regions I and III in Figure 1(a). Thus, in order to prevent the pest outbreak, parameters $\alpha$ and $\beta$ should be carefully chosen to keep the interior equilibria of two subsystems in region IV.

\section{Numerical Analysis}

4.1. Bifurcation Analysis about Inherent Growth Rate $\alpha$. To study the dynamics of the switching ecological model (3) with IPM, the bifurcation diagram about $\alpha$ provides a summary of essential dynamical behavior of system. We discuss the system through numerical simulation as the system cannot be solved explicitly.

The bifurcation diagrams of the system about parameter $\alpha$ in the range $8<\alpha<34$ are shown in Figure 2 . We observed that the system has more complex behavior, including many chaotic bands, chaotic crises, and period windows. As the parameter $\alpha$ increases form 8 to 9.1, we have one-period attractors of host and parasitoid populations. When $\alpha$ increased from 9.1 to 10.9 , the system experiences quasiperiodicity attractors. As $\alpha$ further increases, 5-piece attractors generate at $\alpha=11$, and we see pitchfork bifurcation with period-doubling cascade to 10-piece attractors at $\alpha=$ 13.1. When $\alpha$ is between 15 and 17.5, the system goes through very complicated behavior, including chaos bands, narrow and wide period windows, and chaos crises. The range when $18<\alpha<21.2$ represents the periodic attractors with period16. Subsequently, the system experiences a wide chaotic bands, periodic windows, narrow chaotic bands, and Hopf bifurcation between 21.3 and 26. In particular, there exists a wide multiattractors coexistence region when $\alpha \in(24.8,26)$, and the details on multiattractors coexistence are discussed in the following section. Finally, the system goes through periodic attractors with period-5 in the range $(26,30)$.

We know that the different initial densities of both host and parasitoid populations can result in different dynamics behaviors, especially different outbreak patterns (here outbreak means that the density of host population is larger than ET), which help us to design control strategies and to make management decisions. For example, the bifurcation diagrams have several different attractors which can coexist for a wide range of parameters as shown in Figure $2(\alpha \epsilon$ $(24.8,26))$. Therefore, we will focus on how the initial densities affect the final states or host outbreaks and consequently successful pest control. To know how the initial densities of host and parasitoid populations affect the pest outbreak and control strategies, we fix all parameters as those in Figure 3 and choose different initial densities. For example, four different host-outbreak attractors coexist at $\alpha=25$, from which we can find that the four host-outbreak attractors have the different amplitudes and frequencies. Let the initial density of host and parasitoid be $\left(H_{0}, P_{0}\right)=(7.4,0.6)$; then the outbreak patterns for host population are quite complex, as shown in 


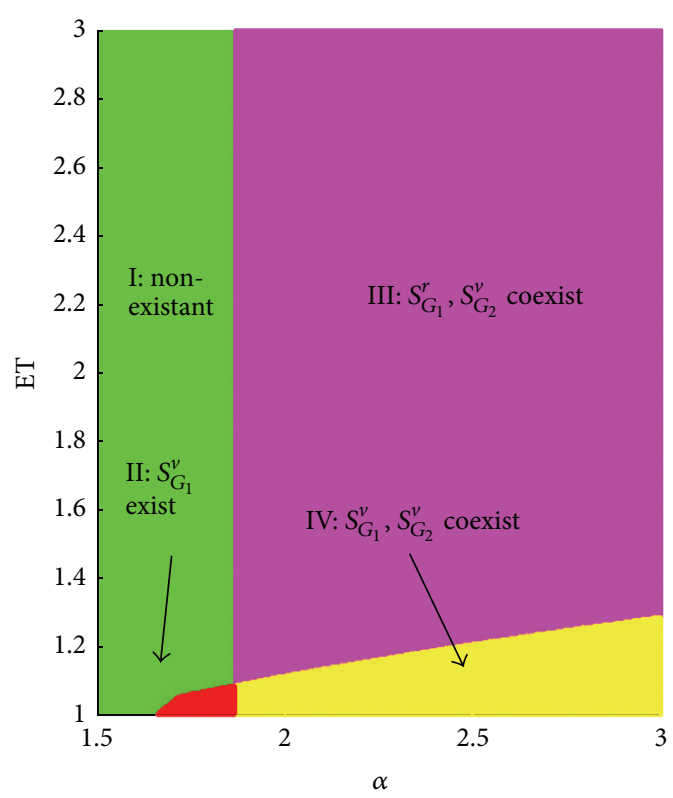

(a)

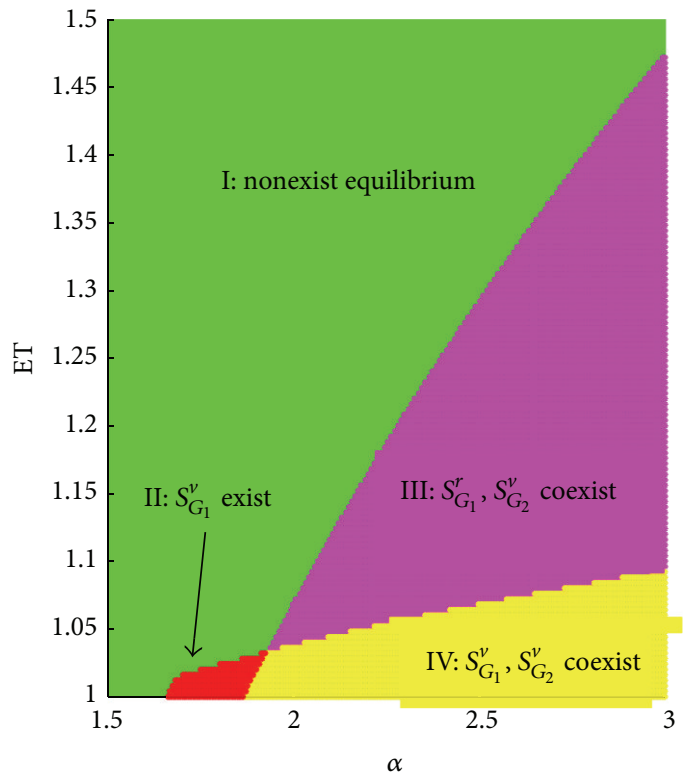

(b)

FIGURE 1: Parameter bifurcation diagram for the existence of equilibria of system (3). Here we choose $\alpha$ and ET as bifurcation parameters and fix others as follows: $P=0.1, k=1, \tau=0.5$. (a) $\alpha \in[1.6,2.9]$, ET $\in[1,3]$, and $\beta=3$; (b) $\alpha \in[1.6,2.9]$, ET $\in[1,3]$, and $\beta \in[1,4]$, simultaneously. Region I (green): nonexistent equilibria; region II (red): $S_{G_{1}}^{v}$ exist; region III (purple): $S_{G_{1}}^{r}$ and $S_{G_{2}}^{v}$ coexist; region IV (yellow): $S_{G_{1}}^{v}$ and $S_{G_{2}}^{v}$ coexist.
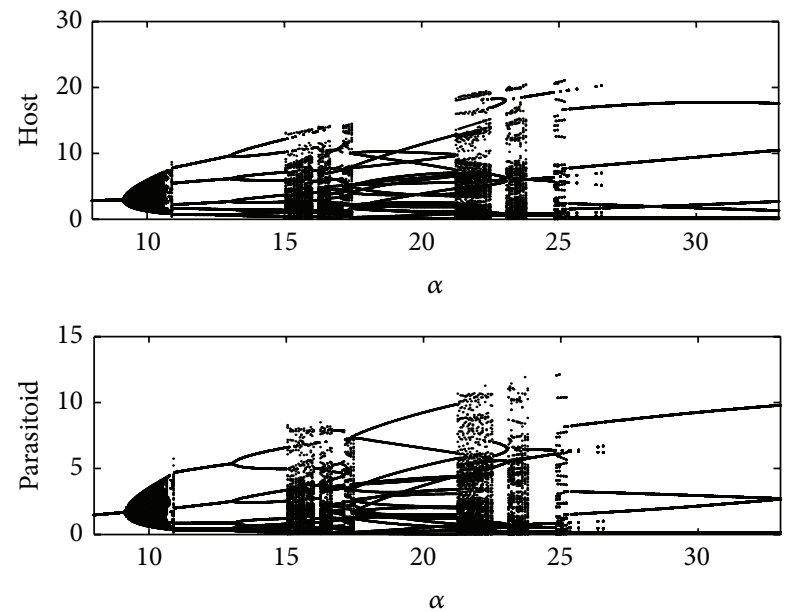

FIgURE 2: Bifurcation diagrams of model (3). For each $\alpha$ the first 1101 simulated values are omitted to remove the initial transients and only the next 100 values are plotted. The host and parasitoid populations are plotted for 500 values of $\alpha$ over $[8,34]$. The other parameters are fixed as $p=0.65, \mathrm{ET}=7.5, \beta=0.5, \tau=2, \alpha=$ $12, b=1$.

Figure 3(a); the maximal host-outbreak amplitude (density) is 20.5998, and the system experiences 25 generation and reach the next maximal outbreak (e.g., the first maximal outbreak lies in 1408 generation, and the next maximal outbreak lies in 1433 generation). Moreover, between 1408 generation and 1433 generation, we can see that the system experiences four different smaller amplitude outbreaks (in other words, these amplitudes are greater than ET, less than the maximal amplitude 20.5998), which lie in 1413, 1418, 1422, and 1428 generation, respectively. If we let the initial values be $\left(H_{0}, P_{0}\right)=(6,2)$, then the maximal host outbreak density of the system (3) is 20.816 (see Figure 3(c)), and the attractor oscillates with period 20 (e.g., the first maximal outbreak lies in 1408 generation, and the next maximal outbreak lies in 1428 generation). Note that, this attractor shows that it will go through three different smaller amplitude outbreaks between 1408 generation and 1428 generation (which lies in 1413, 1418, and 1423 generation, resp.). The third attractor has the same host outbreak amplitudes which is 16.5735 and the outbreak frequencies with period 5 , where the initial value is $\left(H_{0}, P_{0}\right)=$ $(6.2,4.5)$, as shown in Figure 3(e). The fourth attractor has the same host outbreak frequencies as the third attractor, where the initial value is $\left(H_{0}, P_{0}\right)=(6,4.8)$. However, the outbreak amplitudes which are 19.3653 are higher than the third attractor, as shown in Figure 3(g).

By comparing these four attractors, we conclude that different host-parasitoid initial densities may result in different host-outbreak solutions firstly. The first and second attractors have very complex outbreak amplitudes, which are shown in Figures 3((a)-(b)) and 3((c)-(d)), respectively. It is very difficult to design control strategy to prevent pest outbreak. Moreover, the third attractor and the forth attractor have the same outbreak frequency and the unique outbreak amplitudes, as shown in Figures 3((e)-(f)) and 3((g)-(h)); the pest outbreak is easy to control. In particular, the third attractor is better than the forth, because the amplitudes are 


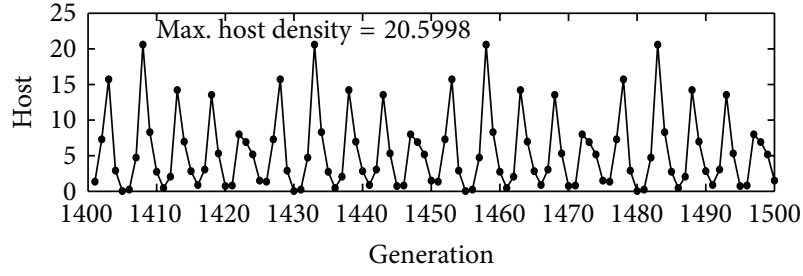

(a)

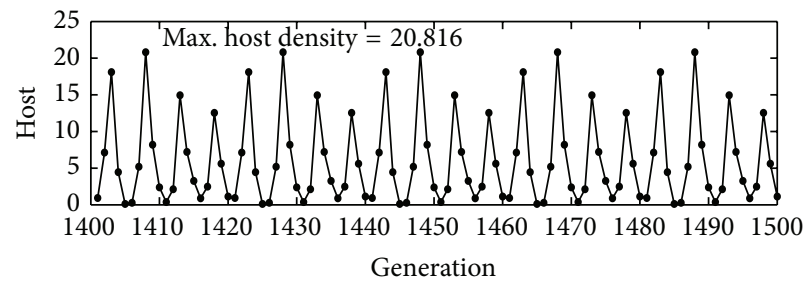

(c)

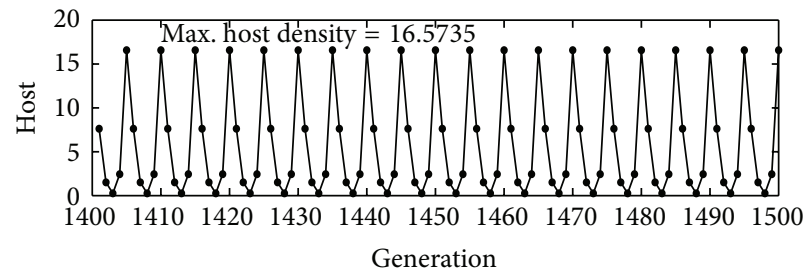

(e)

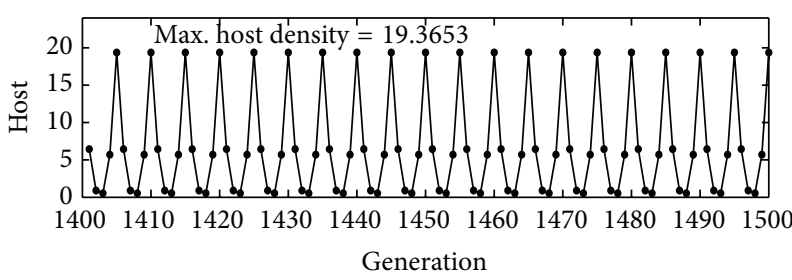

(g)

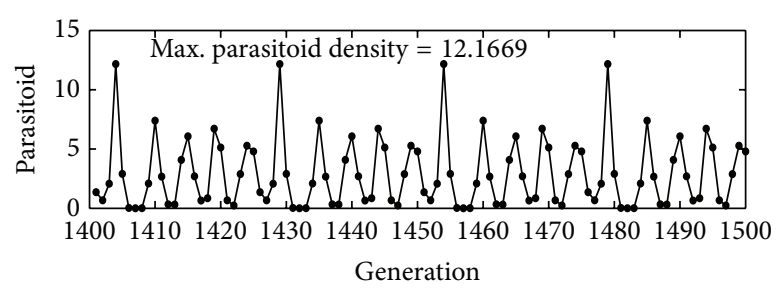

(b)

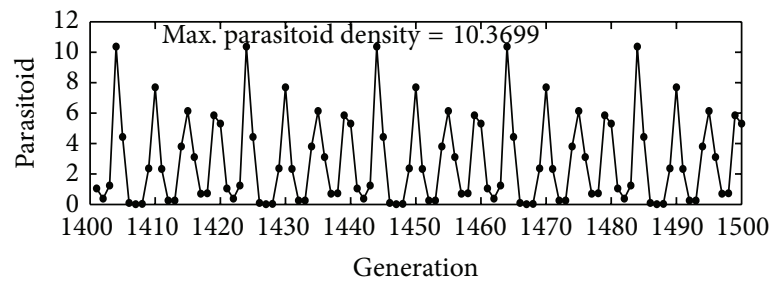

(d)

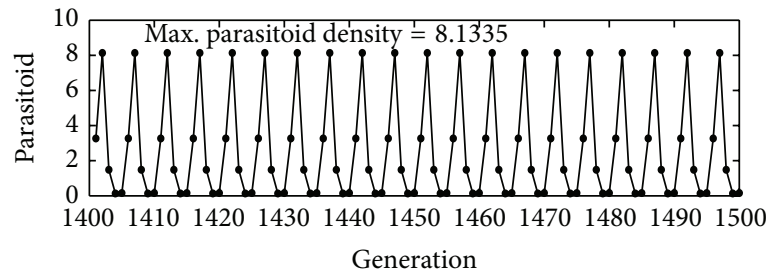

(f)

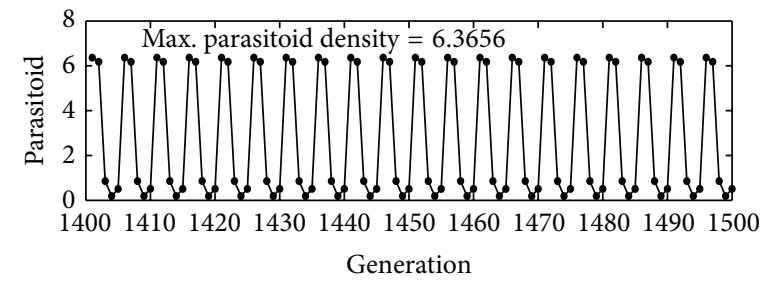

(h)

FIGURE 3: Four coexisting attractors of system (3) with parameters $p=0.65$, ET $=7.5, \beta=0.5, \tau=2, \alpha=25, b=1$. The initial conditions of $((\mathrm{a})-(\mathrm{b})),((\mathrm{c})-(\mathrm{d})),((\mathrm{e})-(\mathrm{f}))$, and $((\mathrm{g})-(\mathrm{h}))$ are $\left(H_{0}, P_{0}\right)=(7.4,0.6),(6,2),(6.2,4.5)$, and $(6,4.8)$, respectively. ((a)-(b)) Periodic attractor with period 25, ((c)-(d)) periodic attractor with period 20, ((e)-(f)) periodic attractor with period 5, and ((g)-(h)) periodic attractor with period 5.

smaller. Thus, for the first, second, and forth attractors, we can change the initial value of parasitoid density (releasing or harvesting the natural enemies) such that the system changes into the third attractor as in Figures 3(e) and 3(f). In the next section, we will discuss the initial value region of the host and parasitoid.

In order to control the host population successfully such that its density decreases and falls below ET, the initial densities of both host and parasitoid populations should be monitored and tracked carefully. The basins of attraction shown in Figure 4 indicate that the four attractors can coexist (see Figure 3) in a various host-parasitoid initial densities. The horizontal axis and vertical axis are the host and parasitoid initial values, respectively. In Figure 4(a), the initial value ranges are $0 \leq H_{0} \leq 16,0 \leq P_{0} \leq 8$ and Figure 4(b) is an enlargement of Figure 4(a) with range $0 \leq$ $H_{0} \leq 8,0 \leq P_{0} \leq 8$. There exist the basins of attraction for four host attractors: the magenta, blue, green, and yellow areas are the attraction regions for the periodic solutions shown in Figures 3((a)-(b)), 3((c)-(d)), 3((e)-(f)), and 3((g)(h)), respectively. Thus, we conclude that the green area may be an ideal initial area for host control according to analysis of coexistence attractors shown in Figure 3. Moreover, we can find the fractal properties of the self-similarity and fractal basin boundaries. Note that the basins of attraction separate the attraction regions into two parts by the line $H_{0}=$ ET (here ET $=7.5$ ), which reveal that there exist different hostoutbreak patterns and control strategies.

4.2. Bifurcation Analysis about Insecticidal Rate p. The most effective strategy for controlling pests may be to combine methods in an approach known as integrated pest management (IPM) that emphasizes the prevention of pest damage. If all other integrated pest management tactics are unable to keep an insect pest population below an economic threshold, then the use of an insecticide to control the pest and prevent 


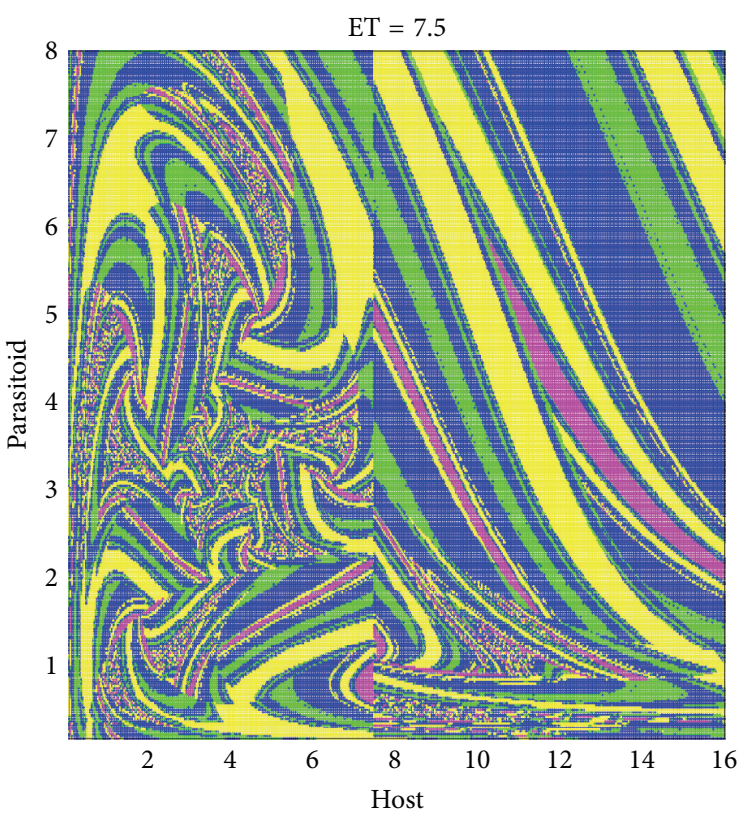

(a)

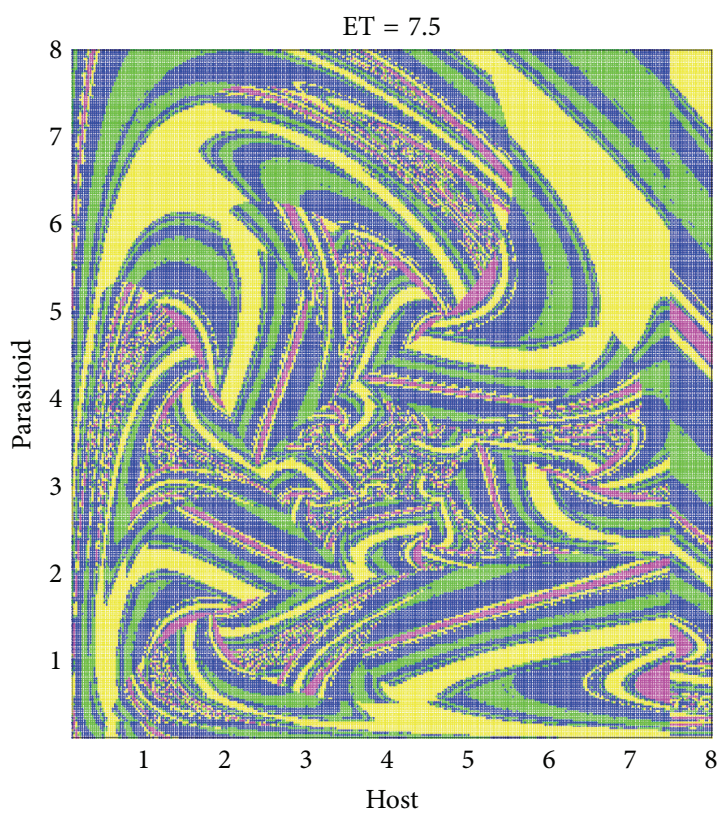

(b)

FIGURE 4: Basins of attraction of four coexisting attractors of system (3). The horizontal axis and vertical axis are the host and parasitoid initial values $H_{0}, P_{0}$, respectively. (a) Basin of attraction of four periodic solutions shown in Figure 3, where the intervals of initial values are $0 \leq H_{0} \leq 16,0 \leq P_{0} \leq 8$; (b) an enlargement of (a) with range $0 \leq H_{0} \leq 8,0 \leq P_{0} \leq 8$. The other parameters are fixed the same as in Figure 3. The magenta region represents Figures $3(\mathrm{a})$ and $3(\mathrm{~b})$ attractor, the blue region represents Figures $3(\mathrm{c})$ and $3(\mathrm{~d})$ attractor, the green region represents Figures 3(e) and 3(f) attractor, and the yellow region represents Figures 3(g) and 3(h) attractor.
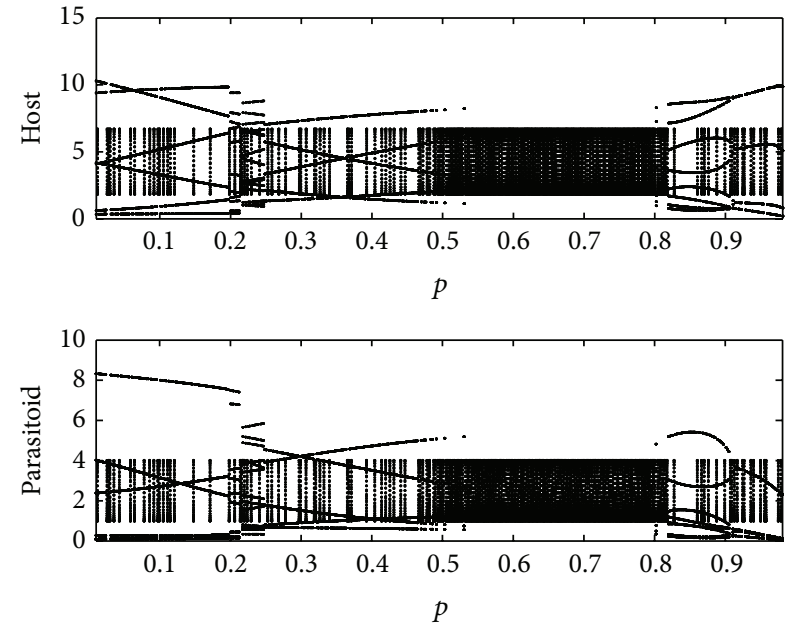

FIGURE 5: Bifurcation diagrams of model (3). For each $p$ the first 1101 simulated values are omitted to remove the initial transients and only the next 100 values are plotted. The host and parasitoid populations are plotted for 500 values of $p$ over $[0.01,0.99]$. The other parameters are fixed as ET $=6.73, \tau=2, \alpha=12, b=1$, $\left(H_{0}, P_{0}\right)=(3,1)$.

economic loss is justified. Spraying insecticides is a very effective strategy for pest control. In this section, we discuss the impact of insecticidal rate on pest outbreaks. As we will show in Figure 5, the killing rate of pesticides $p$ also acts as an important factor for host control. It follows from the bifurcation diagram that the host population can outbreak for small and large killing rate of pesticides (e.g., $p \in(0.01,0.59)$ and $(0.78,0.97))$. Moreover, Figure 5 clearly shows how to choose suitable killing rate such that the whole system can stabilize in the subsystem $S_{G_{1}}$ and provide a possible explanation on the Volterra principle. If we carefully choose the killing rate (e.g., $p \in[0.6,0.78]$ ), then the host population can stabilize in the subsystem $S_{G_{1}}$.

To confirm this, we choose $p=0.61$; the other parameter is fixed as those in Figure 6. The results show that there exist four different cases in which host population can eventually stabilize below an economic threshold. Figure 6(a) shows that the pest (host) density never reaches the given ET (here ET = 0.63, $\left.\left(H_{0}, P_{0}\right)=(4,3)\right)$, which confirms that the solution initiating from $(4,3)$ is free from IPM tactics. If we set the initial densities as $(6,6),(3,4.5)$, or $(4,5)$, the results indicate that the system is eventually free after one, two, or three times when chemical and biological IPM tactics are applied (see Figures 6(b), 6(c), or 6(d)). All those confirm that the stability of interaction between host and parasitoid plays a very important role in pest control, and the control tactics should be designed under this rule.

Figure 6 shows that the system will eventually stabilize in the $S_{G}$ subsystem in different cases. Thus, the initial densities of both host and parasitoid populations should be monitored and tracked carefully. We further discuss the above cases on the host densities and the parasitoid densities plane (see Figure 7(a)), which shows that there exist four different regions which are denoted by I, II, III, IV, and V with different 


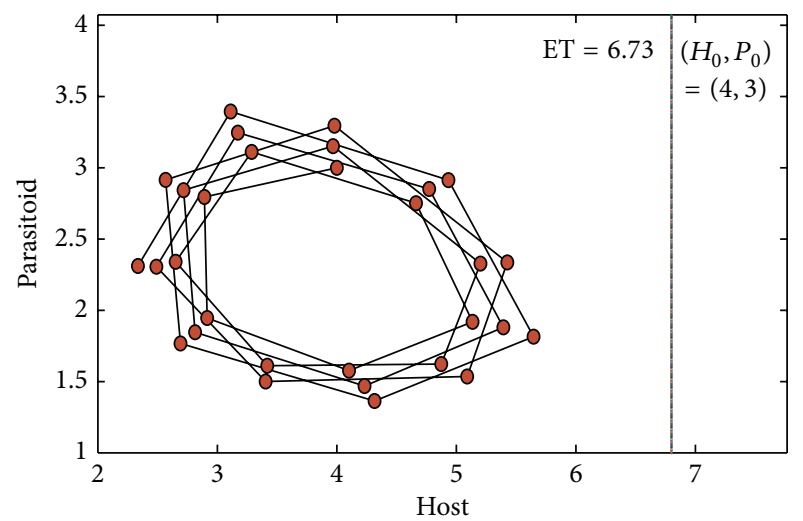

(a)

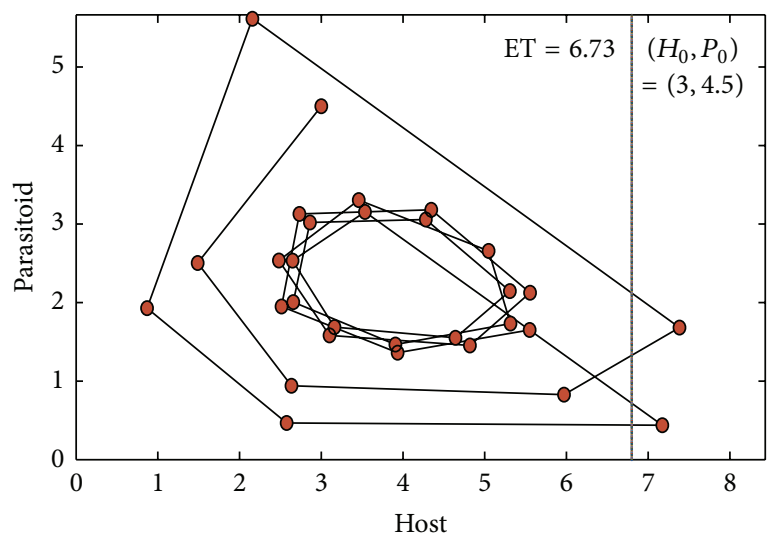

(c)

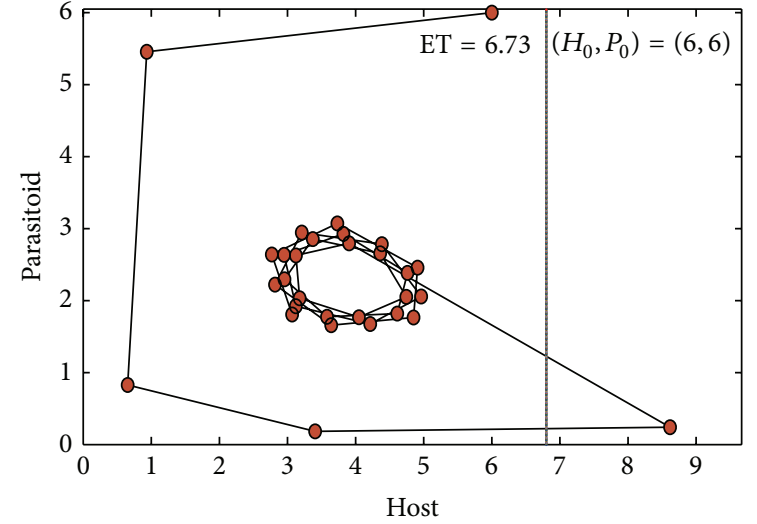

(b)

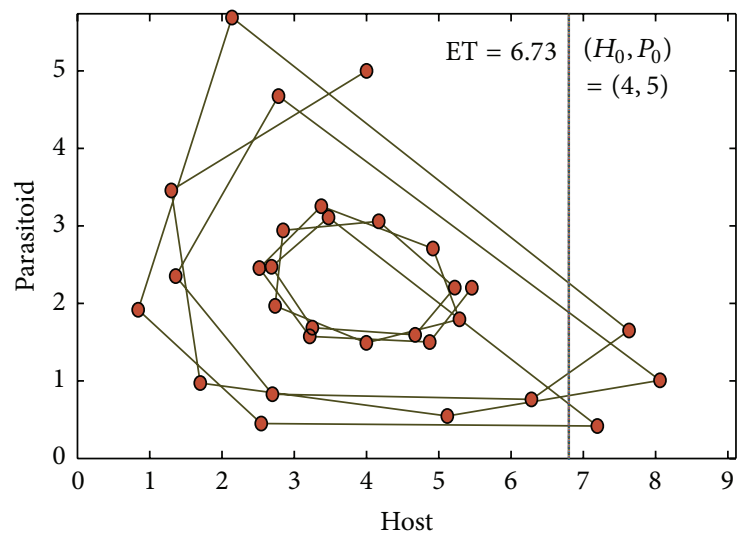

(d)

FIGURE 6: Illustrating the switch effects of initial densities of the host and parasitoid populations of model (3) on IPM. The parameters are fixed as $p=0.61, \mathrm{ET}=6.73, \beta=0.4, \tau=2, \alpha=12, b=1$. The initial densities $\left(H_{0}, P_{0}\right)$ in $(\mathrm{a})-(\mathrm{d})$ are $(4,3),(6,6),(3,4.5)$, and $(4,5)$, respectively.

colors. Region I is denoted by yellow; the pest population never outbreaks and always stabilizes in the subsystem $S_{G_{1}}$. In this region, the corresponding case is shown in Figure 6(a). In regions II, III, or VI, the system is free from IPM control after one, two, or three IPM applications (e.g., Figures 6(b), $6(\mathrm{c})$, or $6(\mathrm{~d})$ ); they are marked with green, purple, or red, respectively. In particular, if the killing rate of pesticides decreases slightly (here $p=0.60$ ), the host densities and the parasitoid densities plane are shown as in Figure $7(\mathrm{~b})$. The results show that there emerges a new blue region denoted by $\mathrm{V}$ except for the above four regions. In this new region, the host population periodic outbreaks and can never stabilize the subsystem $S_{G_{1}}$ subsystem. Through the above analysis, we can see that the killing rate of pesticides is highly sensitive to host population stabilize in the subsystem $S_{G_{1}}$, and the blue region is unfavorable.

Having looked at pest outbreak frequencies, we now turn to a study of the different killing rates of the same system. To compare the effect of the different killing rates of pesticides, we analyze the pest (host) outbreak times of initial densities of both host and parasitoid populations for different killing rate as shown in Figures $8(\mathrm{a})-8(\mathrm{~d})$. The results show that they have the same yellow region where the pest population never outbreaks. This is a very important phenomenon for pest control. For example, if we let initial densities of host and parasitoid lie in this yellow region, the host population will never outbreak. However, for the regions which have one, two, three, or many outbreak times, the different killing rate has a great impact on the pest outbreak region. For example, if we let $p$ be small (here $p=0.35$ ), there exists a large blue area where pest (host) populations experience many times outbreak and very small green and purple region where the host population can stabilize from IPM control after one and two IPM applications, as shown in Figure 8(a). If we increase $p=0.6$, Figure 8 (b) shows that the green and purple region areas are rapidly expanded, the blue region area is reduced, and a new red region area where host population experiences three times outbreak is emerged. If we further increase $p=$ 0.75, as shown in Figure 8(c), the blue region area becomes larger than that in Figure 8(b). Moreover, if we let $p=0.85$, the whole region is divided into two parts (blue region and yellow region) and the red and purple regions disappear (see Figure 8(d)).

In short, these results confirm that different host-parasitoid initial densities may result in different final stats of host population. Moreover, the different killing rate of 


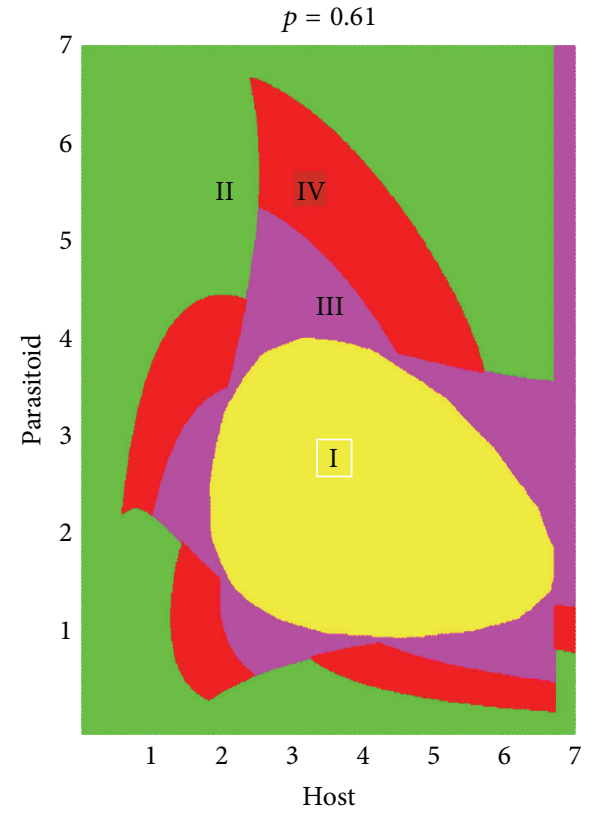

(a)

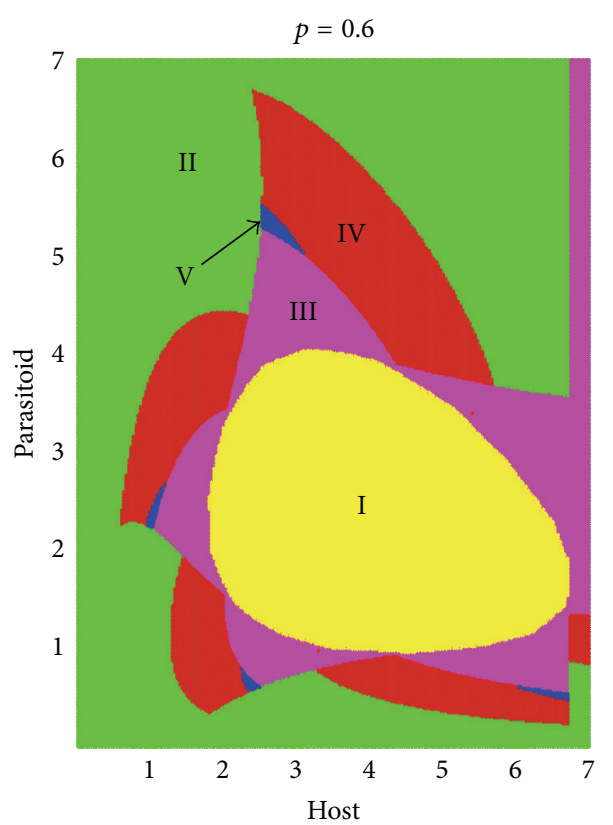

(b)

FIGURE 7: Illustrating how the pest outbreak frequencies depend on initial densities of the host and parasitoid populations of model (3) on IPM. The parameters are fixed as ET $=6.73, \beta=0.4, \tau=2, \alpha=12, b=1$. Region I, II, III, IV, and V are denoted without pest outbreak by one, two, three, and many times pest outbreak, respectively. (a) $p=0.61$. (b) $p=0.6$.

pesticides can result different final sates region. If we select the right killing rate of pesticides (see Figure 6(a)), the host population which go through one, two or three IPM applications can finally stabilize in the subsystem $S_{G_{1}}$.

\section{Conclusion}

It is well known that frequently using pesticides kills pests effectively but may cause serious environmental problems and result in faster development of drug resistance. The integrated pest management as an approach to control insect pests must be committed to a long-term strategy which includes biological control (releasing natural enemies or harvesting pest insets) and chemical control (spaying pesticides). In order to keep the density of pest population below the economic threshold or prevent the pest outbreak, the effective monitoring must be done to keep track of the pest populations. In other words, once the density of pest population exceeds the economic threshold, the IPM application should be adopted. We have extended the discrete hostparasitoid model with pest Beverton-Holt growth concerning IPM strategy and constructed the switched discrete hostparasitoid model which are composed of the free subsystem and controllable subsystem, and the switches are guided by the economic threshold in this paper.

To understand the switching model interaction among biological control, chemical control, and economic threshold, we firstly discuss the existence of the interior equilibria of subsystem $S_{G_{1}}$ and subsystem $S_{G_{2}}$ and the equilibria of the whole switching system (3). By extensive numerical bifurcation investigations about parameter $\alpha$ in the range $8 \leq \alpha \leq 34$ (see Figure 2), we find that the system has more complex behavior, including many chaotic bands, chaotic crises, and period windows. In addition, multiple attractors from which the pest population oscillates with different amplitudes can coexist for a wide range of parameters $(\alpha \in[24.8,26])$. For example, four different host-outbreak attractors coexist at $\alpha=25$ as shown in Figure 3, from which we can see that the four host-outbreak attractors coexist with the different amplitudes and frequencies. The results indicate that the dynamic behavior of a population may be dramatically affected by small change in the values of initial densities of the host and parasitoid populations. The basins of attraction shown in Figure 4 indicate that the four attractors can coexist in a various host-parasitoid initial densities. There exist the basins of attraction for four host attractors: the magenta, blue, green, and yellow areas are the attraction regions for the periodic solutions shown in Figures 3((a)(b)), 3((c)-(d)), 3((e)-(f)), and 3((g)-(h)), respectively. Thus, we conclude that the green area may be an ideal area for host control according to analysis of coexistence attractors shown in Figure 3. Moreover, the basins of attraction separate the attraction regions into two parts by the line $H_{0}=\mathrm{ET}$ (here ET $=7.5$ ), which reveal that there exist different hostoutbreak pattern and control strategies.

In fact, the killing rate of pesticides $p$ is also an important factor for host control. By extensive bifurcation investigations about parameter $p$ (see Figure 5), it is verified that the system can eventually stabilize in the subsystem $S_{G_{1}}$ on condition that we choose the appropriate killing rate (e.g., $p=0.61$ ). Figure 6 shows that there exist four different cases in which host population can eventually stabilize below the ET after 


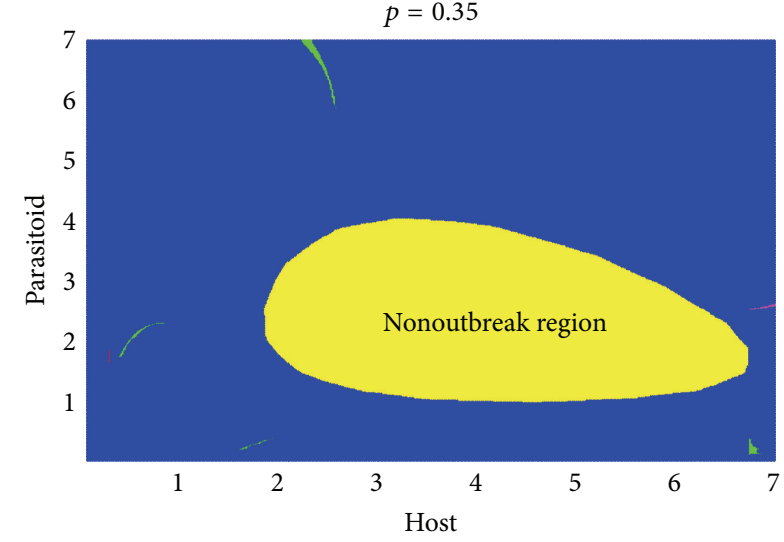

(a)

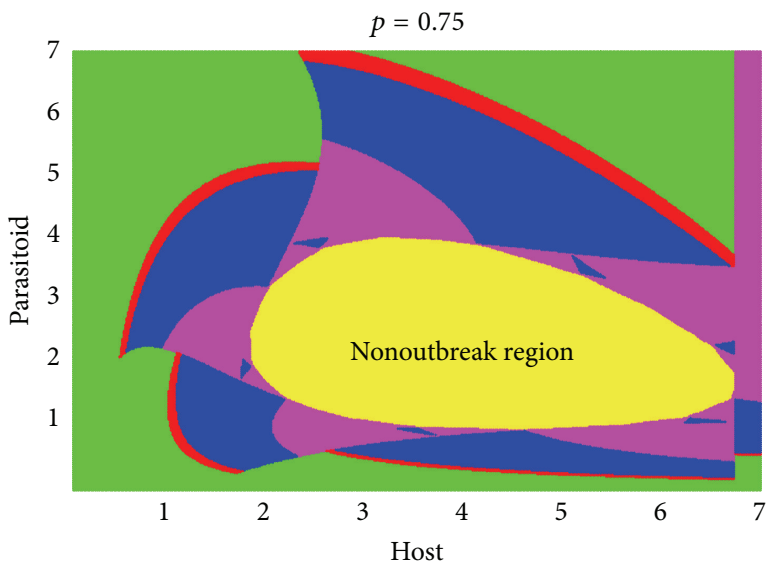

(c)

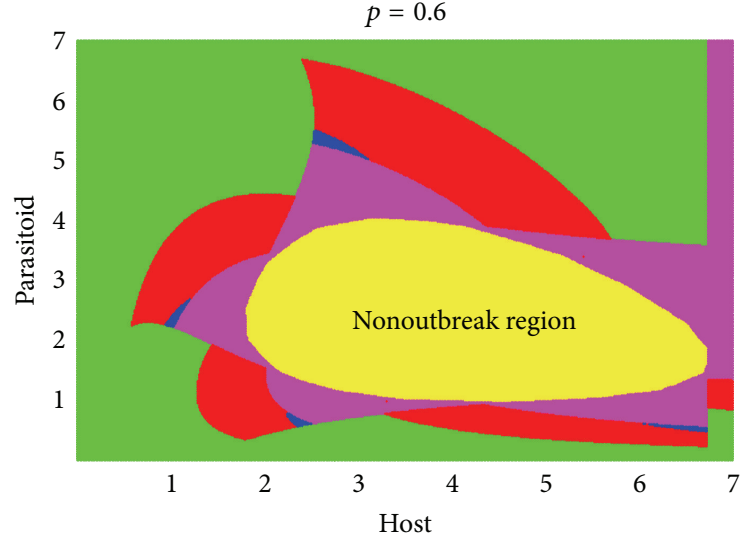

(b)

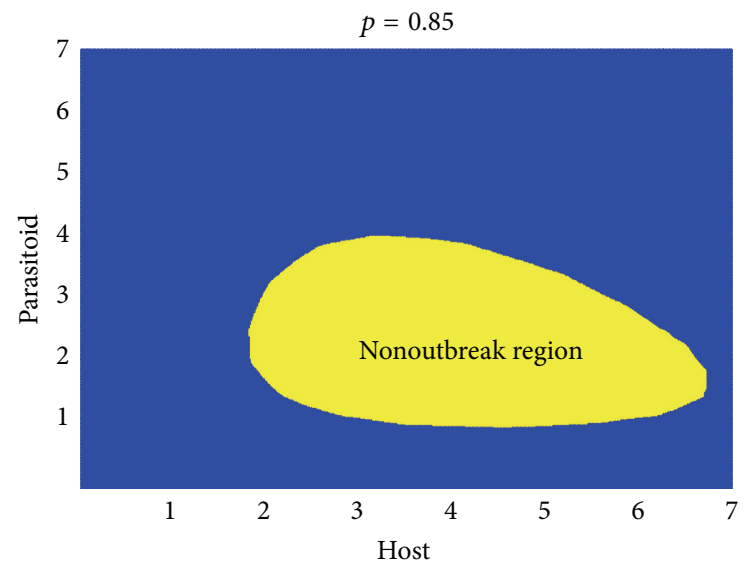

(d)

FIGURE 8: Compare the effect of the different killing rate of pesticides on pest outbreak frequencies. The other parameters are fixed as ET $=$ 6.73, $\beta=0.4, \tau=2, \alpha=12, b=1$. (a) $p=0.35$; (b) $p=0.6$. (c) $p=0.75$. (d) $p=0.85$. Different color regions corresponding to Figure 7 .

zero, one, two, or three times when IPM tactics are applied, respectively. In the initial density plane of host population and parasitoid population, there exist four different regions which represent the different outbreak times. Moreover, the killing rates have great impact to these regions as shown in Figure 8. It is shown that the different killing rate of pesticides can result in different final regions. If we select the right killing rate of pesticides (see Figure 6(a)), the host population which experiences one, two, or three IPM applications can finally stabilize in subsystem $S_{G_{1}}$. These phenomena provide important references for IPM applications.

This work focused entirely on the simplest host-parasitoid model with pest Beverton-Holt growth concerning IPM strategy and the temporal interactions of an insect host and its parasitoid. The spatial heterogeneity or density-dependent growth of the host and functional responses of the parasitoid will be considered in the coming research.

\section{Conflict of Interests}

The authors declare that there is no conflict of interests regarding the publication of this paper.

\section{Acknowledgments}

This work is supported by the Key Laboratory of Biologic Resource Protection and Utilization of Hubei province (no. PKLHB1332), the Natural Science Foundation of Hubei province (no. CDZ2010047), and the Soft Science Research Project of Hubei province (2012GDA01309).

\section{References}

[1] S. Tang and R. A. Cheke, "Models for integrated pest control and their biological implications," Mathematical Biosciences, vol. 215, no. 1, pp. 115-125, 2008.

[2] S. Tang, Y. Xiao, and R. A. Cheke, "Multiple attractors of hostparasitoid models with integrated pest management strategies: eradication, persistence and outbreak," Theoretical Population Biology, vol. 73, no. 2, pp. 181-197, 2008.

[3] Y. Xiao and F. van den Bosch, "The dynamics of an eco-epidemic model with biological control," Ecological Modelling, vol. 168, no. 1-2, pp. 203-214, 2003.

[4] F. D. Parker, "Management of pest populations by manipulating densities of both hosts and parasites through periodic releases," 
in Biological Control, pp. 365-376, Springer, New York, NY, USA, 1971.

[5] V. M. Stern, R. F. Smith, R. van den Bosch, and K. S. Hagen, The Integration of Chemical and Biological Control of the Spotted Alfalfa Aphid, University of California, Berkeley, Calif, USA, 1959.

[6] J. Liang and S. Tang, "Optimal dosage and economic threshold of multiple pesticide applications for pest control," Mathematical and Computer Modelling, vol. 51, no. 5-6, pp. 487-503, 2010.

[7] J. Liang, S. Tang, R. A. Cheke, and J. Wu, "Adaptive release of natural enemies in a pest-natural enemy system with pesticide resistance," Bulletin of Mathematical Biology, vol. 75, no. 11, pp. 2167-2195, 2013.

[8] S. Tang, J. Liang, Y. Tan, and R. A. Cheke, “Threshold conditions for integrated pest management models with pesticides that have residual effects," Journal of Mathematical Biology, vol. 66, no. 1-2, pp. 1-35, 2013.

[9] S. Tang, J. Liang, Y. Xiao, and R. A. Cheke, "Sliding bifurcations of Filippov two stage pest control models with economic thresholds," SIAM Journal on Applied Mathematics, vol. 72, no. 4, pp. 1061-1080, 2012.

[10] Y. Xiao, X. Xu, and S. Tang, "Sliding mode control of outbreaks of emerging infectious diseases," Bulletin of Mathematical Biology, vol. 74, no. 10, pp. 2403-2422, 2012.

[11] J. Yang, S. Tang, and R. A. Cheke, "Global stability and sliding bifurcations of a non-smooth Gause predator-prey system," Applied Mathematics and Computation, vol. 224, pp. 9-20, 2013.

[12] X. Zhang and S. Tang, "Filippov ratio-dependent prey-predator model with threshold policy control," Abstract and Applied Analysis, vol. 2013, Article ID 280945, 11 pages, 2013.

[13] R. J. H. Beverton and S. J. Holt, The Theory of Fishing, Sea Fisheries, Their Investigation in the United Kingdom, Edward Arnold, London, UK, 1956.

[14] S. R.-J. Jang, "Allee effects in a discrete-time host-parasitoid model," Journal of Difference Equations and Applications, vol. 12, no. 2, pp. 165-181, 2006.

[15] S. R. J. Jang and J. L. Yu, "Discrete-time hostcparasitoid models with pest control," Journal of Biological Dynamics, vol. 6, no. 2, pp. 718-739, 2012.

[16] P. Landi, F. Dercole, and S. Rinaldi, "Branching scenarios in ecoevolutionary prey-predator models," SIAM Journal on Applied Mathematics, vol. 73, no. 4, pp. 1634-1658, 2013.

[17] S. Tang, Y. Xiao, N. Wang, and H. Wu, "Piecewise HIV virus dynamic model with $\mathrm{CD} 4^{+} \mathrm{T}$ cell count-guided therapy: I," Journal of Theoretical Biology, vol. 308, pp. 123-134, 2012.

[18] T. Zhao, Y. Xiao, and R. J. Smith, "Non-smooth plant disease models with economic thresholds," Mathematical Biosciences, vol. 241, no. 1, pp. 34-48, 2013. 


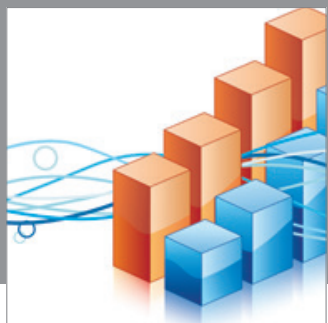

Advances in

Operations Research

mansans

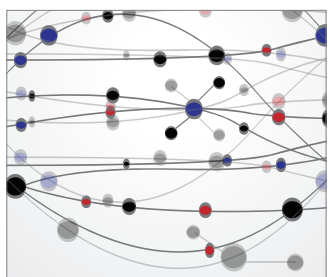

The Scientific World Journal
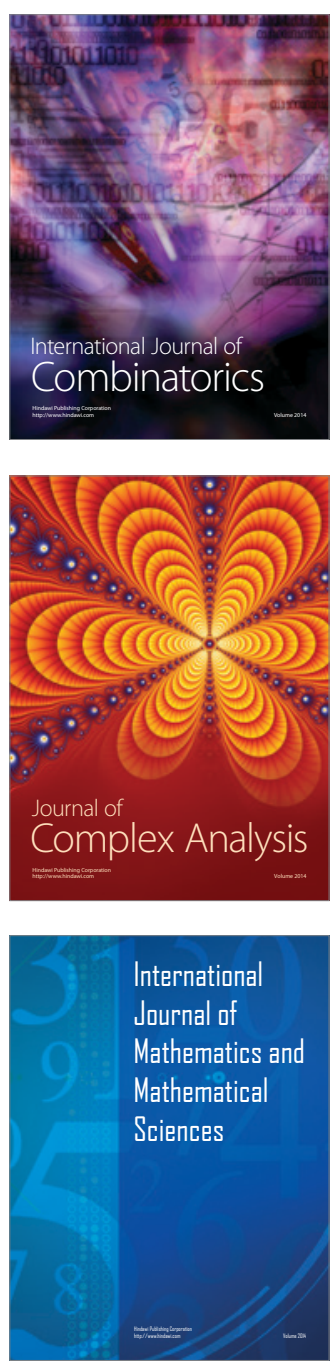
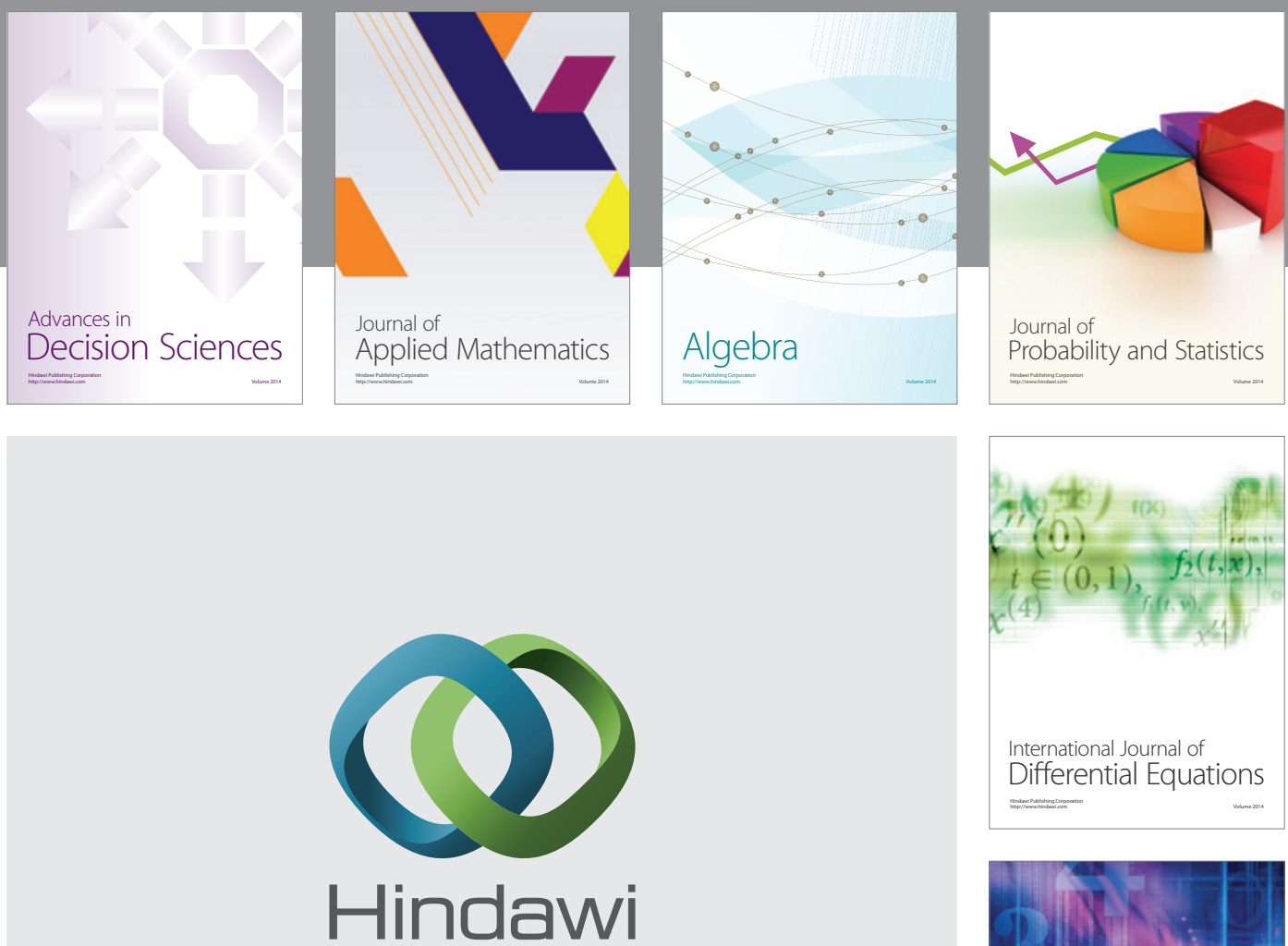

Submit your manuscripts at http://www.hindawi.com
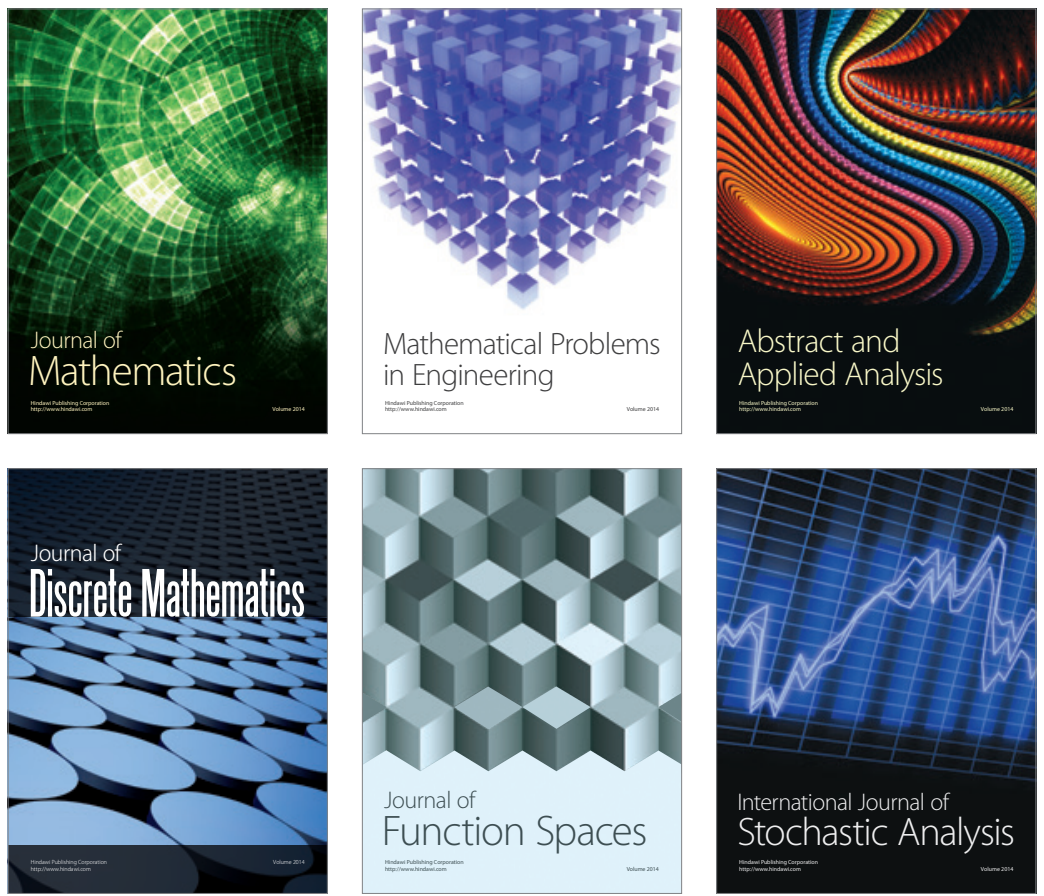

Journal of

Function Spaces

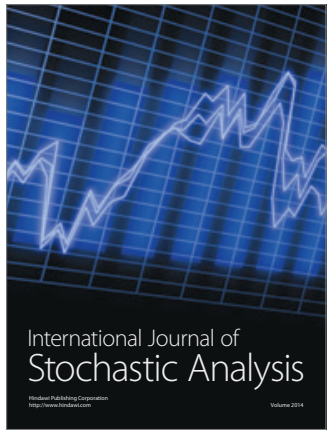

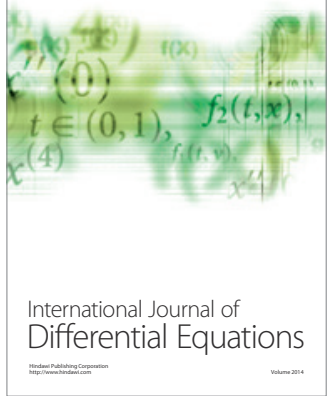
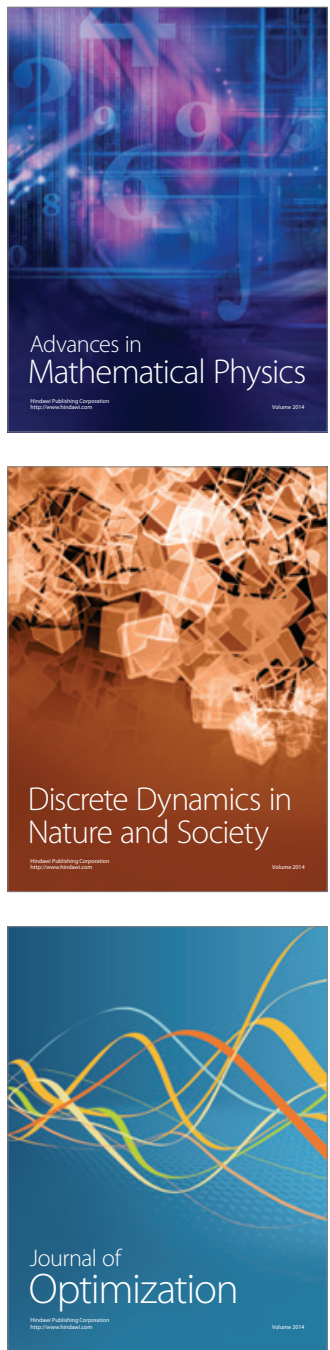